This item was submitted to Loughborough's Research Repository by the author.

Items in Figshare are protected by copyright, with all rights reserved, unless otherwise indicated.

\title{
Hydrodynamic lubricant film separation during co-directional and counter- directional rotations of disengaged wet clutch packs
}

PLEASE CITE THE PUBLISHED VERSION

https://doi.org/10.1115/1.4044443

\section{PUBLISHER}

American Society of Mechanical Engineers

VERSION

AM (Accepted Manuscript)

\section{LICENCE}

CC BY 4.0

\section{REPOSITORY RECORD}

Morris, Nick, Rishi Patel, and Homer Rahnejat. 2019. "Hydrodynamic Lubricant Film Separation During Codirectional and Counter-directional Rotations of Disengaged Wet Clutch Packs". figshare.

https://hdl.handle.net/2134/9159884.v1. 


\section{American Society of Mechanical Engineers}

SETTING THE STANDARD

ASME Accepted Manuscript Repository

Institutional Repository Cover Sheet

ASME Paper Title: Hydrodynamic Lubricant Film Separation During Codirectional and Counter-Directional Rotations

of Disengaged Wet Clutch Packs

Authors: $\quad$ N. J. Morris, R. Patel, H. Rahnejat

ASME Journal Title: Journal of Fluids Engineering

Volume/Issue _142(1)

Date of Publication (VOR* Online) _October 4, 2019

https://asmedigitalcollection.asme.org/fluidsengineering/article/doi/10.1115/1.4044443/956217/

ASME Digital Collection URL: Hydrodynamic-Lubricant-Film-Separation-During

DOI: $\quad$ https://doi.org/10.1115/1.4044443

*VOR (version of record) 


\title{
Hydrodynamic Lubricant Film Separation During Co-Directional and Counter-Directional Rotations of Disengaged Wet Clutch Packs
}

\author{
N.J. Morris*, R. Patel and H. Rahnejat \\ Loughborough University, Loughborough, UK \\ *Corresponding author: $\underline{\text { N.J.Morris@lboro.ac.uk }}$
}

\begin{abstract}
The parasitic drag losses incurred by wet clutches, used in transmission systems, can significantly affect vehicular powertrain efficiency. This paper presents a novel implicit solution for hydrodynamic parasitic drag losses of disengaged clutches. These are generated by conjunctional friction, taking into account lubricant film separation during co-directional and counter-directional disc pair rotations. Lubricant film rupture is considered through application of incipient reverse flow boundary condition, which is representative of lubricant film separation. The results point to the operating conditions at which significant power losses occur. In particular, the time efficient model is able to represent the small losses incurred during co-directional rotation of disc pairs.
\end{abstract}

Keywords: Wet clutch packs, hydrodynamic viscous drag, incipient separation boundary conditions

$\begin{array}{ll}\text { Nomenclature } & \\ h & \text { Integration constants } \\ h_{g} & \text { Axial separation of stator- clutch disc conjunction } \\ h_{p} & \text { Groove axial separation for stator- clutch disc pair } \\ n & \text { Axial separation of stator-clutch disc pad } \\ p & \text { number of Clutch disc grooves } \\ \bar{p} & \text { Pressure } \\ p_{i} & \text { Mean axial pressure across the lubricant film } \\ p_{o} & \text { Boundary pressure at the inner radius } \\ P & \text { Boundary pressure at the free boundary } \\ Q & \text { Power loss } \\ r & \text { Radial volumetric flow rate } \\ r_{c} & \text { Radial position } \\ R_{e} & \text { Radius of curvature } \\ \end{array}$




\begin{tabular}{ll}
$R_{i}$ & Clutch disc inner radius \\
$R_{O}$ & Radial position of free boundary \\
$R e_{c}$ & Reynolds number in the circumferential direction \\
$R e_{r}$ & Reynolds number in the radial direction \\
$w$ & Width of the clutch disc groove \\
$T$ & Drag torque \\
$u$ & Radial velocity \\
$v$ & Tangential velocity \\
$w$ & Fluid velocity in the axial direction \\
$Z$ & Position in the axial direction \\
$Z_{S}$ & Axial location of lubricant film separation \\
$G r e e k$ & \\
$\alpha$ & Surface tension \\
$\Omega_{1}$ & Alow transition factor \\
$\Omega_{2}$ & Angular velocity of the separator disc \\
\hline & Lubricant density \\
\hline &
\end{tabular}

\section{Introduction}

Wet clutch packs are used widely in a variety of applications, including for dual clutch automatic transmissions of commercial passenger vehicles, motorbikes and with transmission systems of offhighway vehicles. In off-highway vehicle transmissions, wet clutches are used to engage and disengage shafts with different drive ratios. Multiple friction discs are inserted onto a male-splined shaft. These friction plates are interspaced by separator plates, retained by the clutch basket so that they would rotate with the secondary shaft. In both, on and off-highway applications a wet clutch system is preferred as the technological solution with high applied torques [1]. This is because the lubricant flow across the friction and separator disc faces mitigate undue thermal, dynamic and wear phenomena $[1,2]$. Unfortunately, the introduction of a viscous medium to the narrow annular conjunction, formed when the clutch pack is disengaged, can generate unwanted parasitic energy losses $[3,4]$. 
The measurement and prediction of wet clutch disengaged performance has attracted considerable attention in literature [5,6,7]. The works of Pahovly et al [5], Iqdal et al [6], Yuan et al [7] and Morris et al [8] have led to a better understanding through the use of analytical models, which consider shear and cavitation between a stationary and a rotating plate. These models typically only consider the motion of one plate, which represents only a portion of the operating conditions experienced in some applications. Similar models have also been suggested by lqbal et al [6], who also considered cavitation through the use of Reynolds, (Swift [9]- Stieber [10]) boundary conditions. They also considered that oil streamers would fill the axial gap between the mating discs through to the outer edge of their contact, satisfying mass flow continuity. Yuan et al [7] used the supply lubricant flow rate and pressure at the inner and outer film radii in order to obtain the position of lubricant film rupture. Morris et al [8] used a separation boundary condition to predict the behaviour of drag losses in disengaged wet brake packs. These models have compared well with experimental measurements.

Commercial computational fluid dynamics software have also been used to investigate the disengaged wet clutch conjunction [11-14]. The works of Mahmud et al [11], Jammulamadaka and Gaokar [12] and Yuan et al [13] have suggested that the lubricant film rupture occurs at a single given radius without the presence of lubricant streamers extending to the conjunctional external radius. The study conducted by Yuan et al [14] showed that the oil film, once ruptured, is swept away by the faster of the two rotating annular surfaces over a cavity of air that extends to the conjunctional external radius. This is supported by the analysis of Zweig and Sneck [15]. Additionally, Yuan et al [14] showed that the rotational speed of both surfaces is critical in determining the position of lubricant film rupture. Indeed, this finding is supported by the experimental observations of Jibin et al [16] and Aphale et al [17] for wet clutches, and also for hydrostatic thrust bearings as demonstrated by Coombs and Dowson [18]. Jibin et al [16] showed through experimental observation that for a single rotating clutch friction disc the oil film once ruptured, is carried away by the rotating surface above the cavity. The predictions and observations made through numerical and experimental work suggest that lubricant film separation rather than cavitation is the critical underlying mechanism for lubricant film rupture in wet clutch packs.

The current paper presents a time efficient analytical model which accounts for both co-directional and counter-directional rotations of friction and separator discs. A lubricant film rupture boundary condition is applied, allowing a single free boundary to be formed at a discrete radius. The expounded analytical model will be of significant use for driveline efficiency modelling, enabling improved understanding of lubricant film behaviour at a variety of friction and separator disc angular velocities.

\section{Analytical Methodology}

The analytical methodology presented here comprises two parts. The first part determines the radial location of lubricant film rupture, whilst the second part deals with the drag losses generated by the coherent lubricant film region which forms between the friction disc's inner radius and the radial location of the rupture boundary. To simplify the problem sufficiently and allow for an implicit analytical solution, a number of assumptions are made. One is to disregard any Poiseuille flow in the tangential direction which may occur in practice due to any disc misalignment. This means that the formation of a convergent-divergent geometry is ignored, which may also be created by the radial cooling channels on the disc. To characterise the combined nature of the radial and circumferential flows, Brunetiére et al [19] presented a combined vector Reynolds numbers, which may be stated as: 
$R e_{c}=\frac{\rho r \Omega \mathrm{h}}{\eta}$

$R e_{r}=\frac{\rho \bar{u} \mathrm{~h}}{\eta}$

Considering laminar flow for: $\alpha<0.56$ [18], then:

$$
\alpha=\sqrt{\left(\frac{R e_{c}}{1600}\right)^{2}+\left(\frac{R e_{r}}{2300}\right)^{2}}
$$

The operational conditions in the current paper determines a laminar lubricant flow regime is maintained. Hou et al [20] found the same finding for comparable operating conditions to those used in this paper.

The current analysis uses a cylindrical co-ordinate frame of reference, where $z$ is the axial distance from the separator disc and $r$ denotes the radial position. The velocities of the fluid in the radial, circumferential and axial directions are represented by $v, u$ and $w$ respectively. For simplicity, when considering the radial location of lubricant film rupture, the conjunction is assumed to be axisymmetric. However, later when predicting the drag torque losses, the influence of radial grooves is included.

First, the location of the free boundary (i.e. that at a constant pressure [21]) must be determined. As already described above, experimental and numerical analyses in open literature indicate that fluid film separates rather than cavitates in the clutch pack conjunctions. Figure 1 is a schematic representation of the lubricant film separation phenomena, also showing the co-ordinate frame of reference used in the current investigation. The angular velocities of the separator disc and friction disc are represented by $\Omega_{1}$ and $\Omega_{2}$ respectively. 


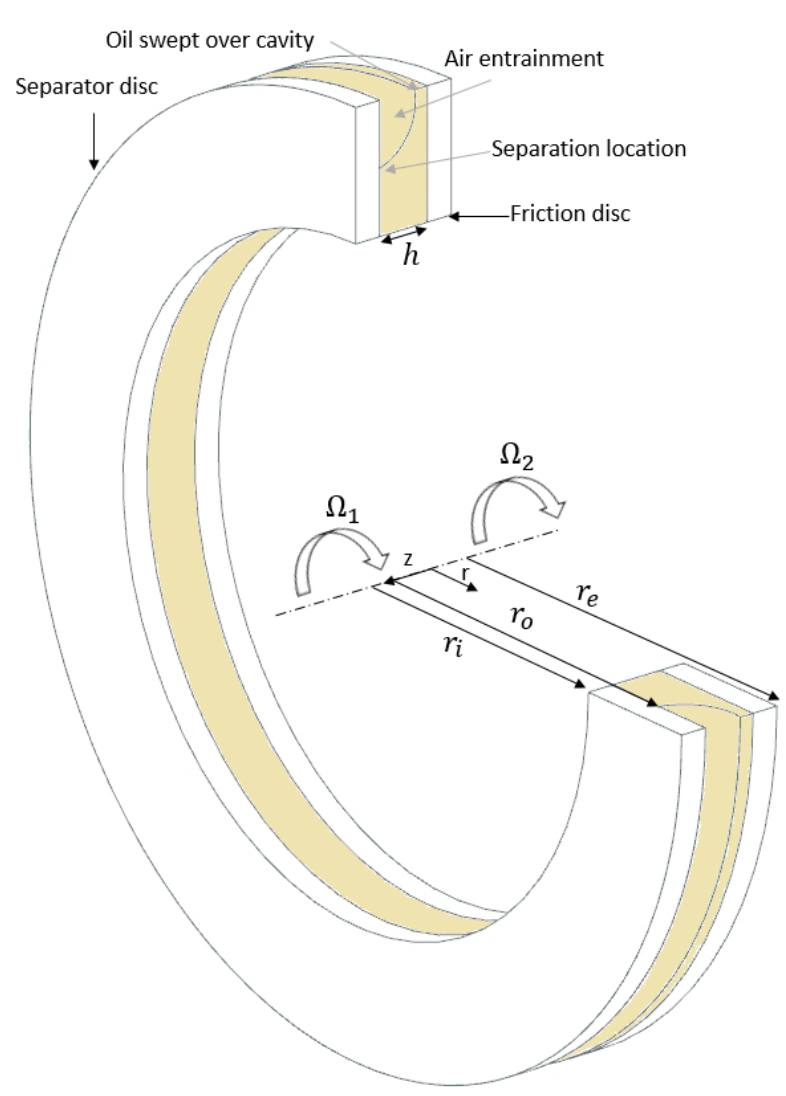

(a)

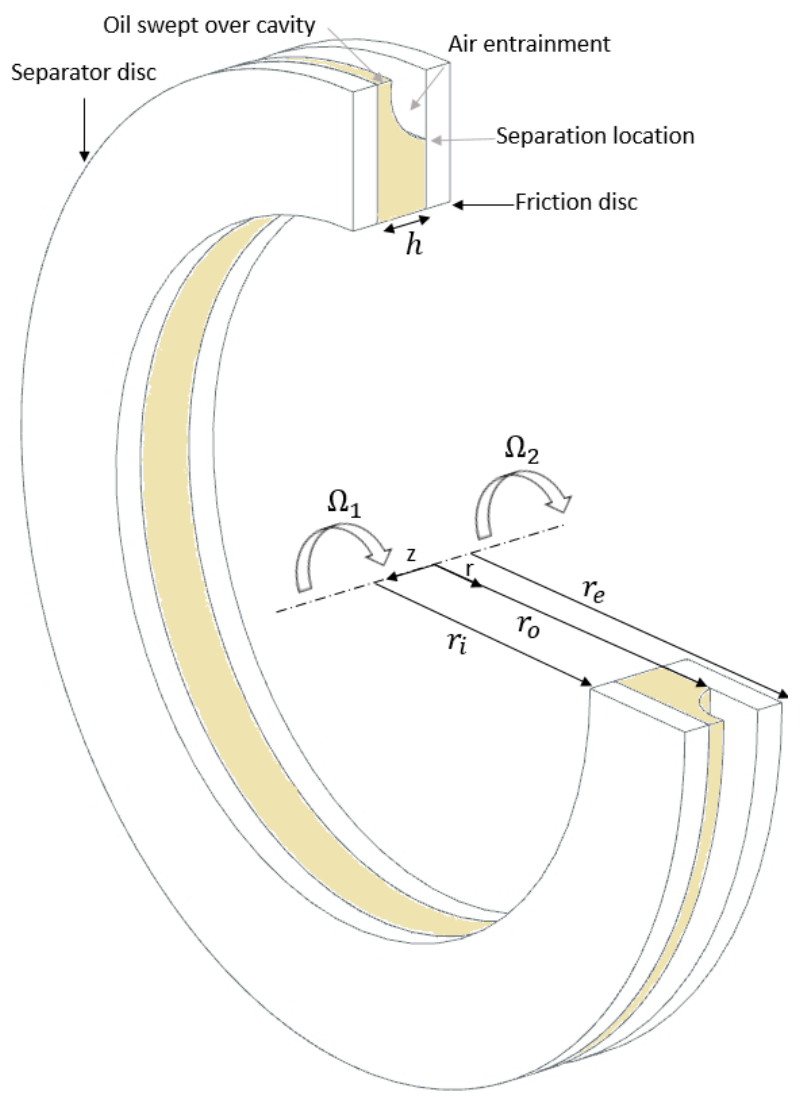

(b)

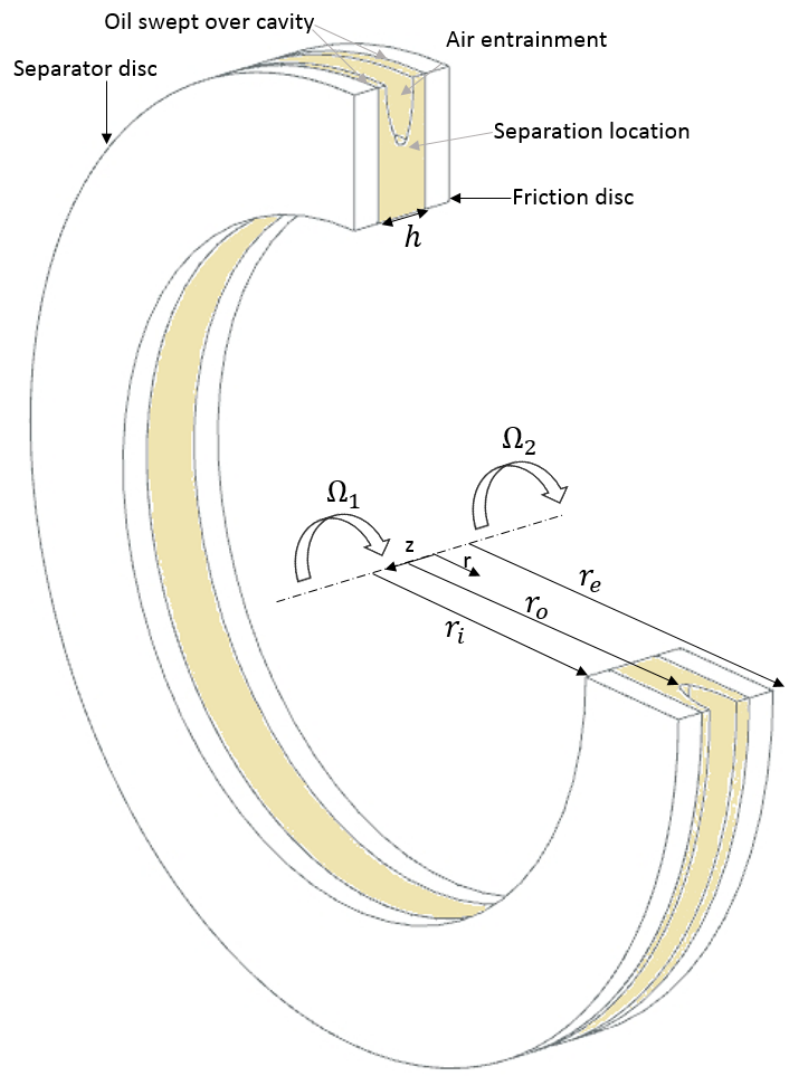


(c)

Figure 1: Sectional diagrammatic representation of disengaged wet clutch contact (not to scale) for the analytical model when (a) $\left|\Omega_{2}\right|>\left|\Omega_{1}\right|$ (b) $\left|\Omega_{1}\right|>\left|\Omega_{2}\right|$ (c) certain instances when $\Omega_{1} \Omega_{2}<0$

Dowson [22] showed that for an assumed axisymmetric conjunction under steady state conditions and considering body forces as negligible, the following equations may be derived from the general Navier-Stokes equations:

$$
\left\{\begin{array}{l}
0=-\frac{\partial p}{\partial r}+\eta \frac{\partial^{2} u}{\partial z^{2}}+\rho \frac{v^{2}}{r} \\
0=\eta \frac{\partial^{2} v}{\partial z^{2}} \\
0=-\frac{\partial p}{\partial z}+\eta \frac{\partial^{2} w}{\partial z^{2}}
\end{array}\right.
$$

The integration of the second of these equations between lower and upper limits of: $z=0, v=\Omega_{1} r$ and $z=h, v=\Omega_{2} r$ yields:

$v=\frac{z}{h} r\left(\Omega_{2}-\Omega_{1}\right)+r \Omega_{1}$

Dowson [22] showed that by using the first and third relations in equation (4) with some manipulation can lead to the following expression:

$\frac{d \bar{p}}{d r}=\eta\left[\frac{\partial^{2} u}{d z^{2}}-\frac{\partial^{2} w}{\partial r \partial z}\right]+\rho \frac{v^{2}}{r}$

As the disc radii is much larger than the plate separation gap, then equations (4), (5) and (6) can be combined to yield an expression for the flow velocity in the radial direction at any axial and radial location:

$u=\frac{z^{2}}{2 \eta} \frac{d \bar{p}}{d r}-\frac{z^{4}}{12 \eta h^{2}} \rho r\left(\Omega_{1}^{2}+\Omega_{2}^{2}\right)+\frac{z^{4}}{6 \eta h^{2}} r \rho \Omega_{2} \Omega_{1}-\frac{z^{3}}{3 \eta h} r \rho\left(\Omega_{2} \Omega_{1}-\Omega_{1}^{2}\right)-\frac{z^{2} r \rho}{2 \eta} \Omega_{1}^{2}+C_{1} z+C_{2}$

Assuming no lubricant film slip at the solid boundaries, the radial flow velocities at each conjunctional surface can be written as: $u=0$ at $z=h$ and at $z=0$. These two boundary conditions can be applied to equation (7) to determine the integration constants $C_{1}$ and $C_{2}$ as:

$C_{2}=0$

And:

$C_{1}=-\frac{h}{2 \eta} \frac{d \bar{p}}{d r}+\frac{h}{12 \eta} \operatorname{\rho r}\left(\Omega_{1}^{2}+\Omega_{2}^{2}\right)-\frac{h}{6 \eta} r \rho \Omega_{2} \Omega_{1}+\frac{h}{3 \eta} r \rho\left(\Omega_{2} \Omega_{1}-\Omega_{1}^{2}\right)+\frac{h}{2 \eta} r \rho \Omega_{1}^{2}$

When the constants of integration are substituted back into equation (7), the expressions for radial velocity gradients and the radial flow velocity can be obtained as:

$$
\begin{aligned}
& \frac{\partial u}{\partial z}=\frac{\left(z-\frac{h}{2}\right)}{\eta} \frac{d \bar{p}}{d r}+\frac{\left(\frac{h^{3}}{4}-z^{3}\right)}{3 \eta h^{2}} \rho r\left(\Omega_{1}^{2}+\Omega_{2}^{2}\right)+\frac{\left(z^{3}-\frac{h^{3}}{4}\right)}{3 \eta h^{2}} r \rho \Omega_{2} \Omega_{1}+\frac{\left(\frac{h^{2}}{3}-z^{2}\right)}{\eta h} r \rho\left(\Omega_{2} \Omega_{1}-\Omega_{1}^{2}\right)+\frac{\left(\frac{h}{2}-z\right) r \rho}{\eta} \Omega_{1}^{2} \\
& u=z(z-h)\left(z^{2} \frac{\rho r}{6 \eta h^{2}}\left(\Omega_{1} \Omega_{2}-\frac{\Omega_{1}^{2}+\Omega_{2}^{2}}{2}\right)+z \frac{r \rho\left(3 \Omega_{1}^{2}-2 \Omega_{1} \Omega_{2}-\Omega_{2}^{2}\right)}{12 \eta h}+\frac{\rho r}{2 \eta}\left(\frac{\Omega_{1} \Omega_{2}}{3}-\frac{2}{3} \Omega_{1}\left(\Omega_{2}-\Omega_{1}\right)-\frac{\Omega_{1}^{2}+\Omega_{2}^{2}}{6}-\Omega_{1}^{2}\right)+\frac{1}{2 \eta} \frac{d \bar{p}}{d r}\right)
\end{aligned}
$$


The continuity of radial flow can be written as:

$\int_{0}^{h} \frac{1}{r} \frac{\partial u r}{d r} d z=0$

Substituting equation (11) into the flow continuity equation (12) yields:

$\frac{\partial}{d r}\left(r \frac{d \bar{p}}{d r}\right)=\frac{3}{5} \rho r\left(\Omega_{1}^{2}+\Omega_{2}^{2}\right)+\frac{4}{5} \rho r \Omega_{1} \Omega_{2}$

Integrating equation (13) with respect to the radius $r$ yields equations (14) and (15), where equation (15) describes the pressure at any radial location in the lubricant film:

$\frac{d \bar{p}}{d r}=\frac{3}{10} \operatorname{\rho r}\left(\Omega_{1}^{2}+\Omega_{2}^{2}\right)+\frac{2}{5} \rho r \Omega_{1} \Omega_{2}+\frac{C_{3}}{r}$

$\bar{p}=\frac{3}{20} \rho r^{2}\left(\Omega_{1}^{2}+\Omega_{2}^{2}\right)+\frac{1}{5} \rho r^{2} \Omega_{1} \Omega_{2}+C_{3} \ln (r)+C_{4}$

To ensure a well-conditioned mathematical problem, three boundary conditions are required. Two of these boundary conditions can be determined as pressure at the internal disc radius and pressure at the free boundary. These can be written as:

$\bar{p}=p_{i}$ at $r=R_{i}$ and $\bar{p}=p_{o}$ at $r=R_{o}$

The pressure at the outer radius can be approximated by the works of Coyne and Elrod $[23,24]$ as:

$p_{o}=-\frac{\sigma}{r_{c}}+\Delta p$

where $r_{c}$ is the radius of curvature of the free boundary film at separation and $\Delta p$ is the pressure change across the transition between the full film and swept film/cavity. When these boundary conditions are applied to equation (15), equations (18) and (19) can be obtained:

$$
\begin{aligned}
& C_{4}=p_{i}-\frac{3}{20} \rho R_{i}^{2}\left(\Omega_{1}^{2}+\Omega_{2}^{2}\right)-\frac{1}{5} \rho R_{i}^{2} \Omega_{1} \Omega_{2}-C_{3} \ln \left(R_{i}\right) \\
& C_{4}=p_{o}-\frac{3}{20} \rho R_{o}^{2}\left(\Omega_{1}^{2}+\Omega_{2}^{2}\right)-\frac{1}{5} \rho R_{o}^{2} \Omega_{1} \Omega_{2}-C_{3} \ln \left(R_{o}\right)
\end{aligned}
$$

A third boundary condition is required to determine the location of the free boundary, $R_{o}$. Birkhoff and Hays [21], Hopkins [25] and Dowson and Taylor [26] have described how ventilated cavities can be formed through fluid film separation in thin lubricating films. This mechanism of film rupture can occur at small sub-ambient pressures, caused by centrifugal flow. It is unlikely that sub-atmospheric pressures for vapours or gaseous cavitation caused by fluid film tension would be encountered in the current application. The separation boundary condition can be written as:

$\frac{d u}{d z}=u=0$

The axial position of separation boundary, $z_{s}$ depends on the angular velocities of the clutch friction disc and the separator plates. Cases 1 and 2 (figure 1 a \&1b) consider the separation condition (equation 20) occurs at the slower of the two rotating conjunctional surfaces. Case 1 specifically accounts for the condition when the oil film separates from the slower moving friction disc surface. Case 2 specifically accounts for the condition when the oil film separates from slower moving 
separator disc surface. These conditions can occur under co-directional rotation of mating surfaces or in their counter-directional rotation, a visual representation of Case 2 and Case 1 is given in figure (1a) and figure (1b) respectively. Case 3 pertains to certain instances of only counter-directional rotation when the condition embodied in equation (20) occurs in the region: $0<z_{s}<h$. A diagrammatic representation Case 3 is shown in figure (1c). The calculation of $z_{S}$ is described later in equation (29). The following solution describes the pressure gradient for film separation at any radius:

\section{(a)- Case 1}

When $\left|\Omega_{1}\right|>\left|\Omega_{2}\right|$, then $\frac{d u}{d z}=u=0$ at $z_{s}=h$

By substituting equation (10) into equation (21), when $r=R_{O}$ the pressure gradient at which the lubricant film separates becomes:

$\frac{d \bar{p}}{d r}=\frac{1}{6} \rho R_{o}\left(3 \Omega_{2}^{2}+2 \Omega_{1} \Omega_{2}+\Omega_{1}^{2}\right)$

Equating (14) and (22), when $r=R_{o}$ yields an expression for the last unknown constant $C_{3}$ for case 1 as:

$C_{3}=\rho R_{o}^{2}\left(\frac{1}{5} \Omega_{2}^{2}-\frac{1}{15} \Omega_{1} \Omega_{2}-\frac{2}{15} \Omega_{1}^{2}\right)$

(b)- Case 2

When $\left|\Omega_{2}\right|>\left|\Omega_{1}\right|$ and $\frac{d u}{d z}=u=0$ at $z_{s}=0$

In a similar manner as previously, substituting equation (10) into equation (24), when: $r=R_{o}$, the pressure gradient at which the lubricant film separates for case 2 becomes:

$\frac{d \bar{p}}{d r}=\frac{1}{6} \rho R_{o}\left(3 \Omega_{1}^{2}+2 \Omega_{1} \Omega_{2}+\Omega_{2}^{2}\right)$

Equating (14) and (25), when $r=R_{o}$, an expression for the last unknown constant $C_{3}$ can be determined for case 2 as:

$C_{3}=\rho R_{o}^{2}\left(\frac{1}{5} \Omega_{1}^{2}-\frac{1}{15} \Omega_{1} \Omega_{2}-\frac{2}{15} \Omega_{2}^{2}\right)$

(c)- Case 3

When $\Omega_{1} \Omega_{2}<0$ and $\frac{d u}{d z}=u=0$ at $0<z_{s}<h$

The axial location and pressure gradient required for $\frac{d u}{d z}=u=0$ to occur away from a surface can be found by determining the single root of the quadratic term in equation (11). This condition only occurs under certain instances of counter-directional rotation (i.e. $\Omega_{1} \Omega_{2}<0$ ).

$Z=\frac{-\frac{r \rho\left(3 \Omega_{1}^{2}-2 \Omega_{1} \Omega_{2}-\Omega_{2}^{2}\right)}{12 \eta h} \pm \sqrt{\left(\frac{r \rho\left(3 \Omega_{1}^{2}-2 \Omega_{1} \Omega_{2}-\Omega_{2}^{2}\right)}{12 \eta h}\right)^{2}-4\left(\frac{r \rho\left(\Omega_{1}^{2}-2 \Omega_{1} \Omega_{2}+\Omega_{2}^{2}\right)\left(\frac{\Omega_{1} \Omega_{2}}{3}-\frac{2 \Omega_{1}\left(\Omega_{2}-\Omega_{1}\right)}{3}-\Omega_{1}^{2}-\frac{\Omega_{1}^{2}+\Omega_{2}^{2}}{6}+\frac{1 d \bar{p}}{2 \eta d r}\right)}{144 \eta^{2} h^{2}}\right)}}{2\left(\frac{r \rho\left(\Omega_{1}^{2}-2 \Omega_{1} \Omega_{2}+\Omega_{2}^{2}\right)}{12 \eta h^{2}}\right)}$ 
The following expression can be obtained as incipient flow occurs when the solution of equation (28) yields a single root:

$z_{S}=\frac{1}{2} \frac{\left(3 \Omega_{1}+\Omega_{2}\right)}{\Omega_{1}-\Omega_{2}} h$

The value of $z_{S}$ can be used to discriminate between cases 1 and 2, and 3. For a single root the pressure gradient can be found as the radical in equation (28) vanishes. Hence:

$\frac{d \bar{p}}{d r}=\frac{1}{24} \rho R_{o}\left(3 \Omega_{1}^{2}+2 \Omega_{1} \Omega_{2}+3 \Omega_{2}^{2}\right)$

Equating (14) and (30), when $r=R_{O}$, an expression for the last unknown constant $C_{3}$ can be determined for case 3 as:

$C_{3}=\rho R_{o}^{2}\left(-\frac{7}{40}\left(\Omega_{1}^{2}+\Omega_{2}^{2}\right)-\frac{19}{60} \Omega_{1} \Omega_{2}\right)$

Finally, through substitution of equations (18) and (19) and either (23), (26) or (31) as appropriate, an expression which can be solved implicitly for each of the operational conditions described by cases 1 , 2 and 3 can be found:

$\frac{p_{i}-p_{o}}{\rho R_{o}^{2} \Omega_{1}^{2}}=\frac{3}{20}\left(\frac{R_{i}^{2}}{R_{o}^{2}}-1\right)\left(1+\frac{\Omega_{2}^{2}}{\Omega_{1}^{2}}\right)+\frac{1}{5}\left(\frac{R_{i}^{2}}{R_{o}^{2}}-1\right) \frac{\Omega_{2}}{\Omega_{1}}+\frac{C_{3}}{\rho R_{o}^{2} \Omega_{1}^{2}} \ln \left(\frac{R_{i}}{R_{o}}\right)$

This equation, which has been rearranged to leave the Euler number of the left-hand side can be solved iteratively to find the value of $R_{o}$ which satisfies the equation. If the value of the outer radius exceeds the external radius (i.e. $R_{o}>R_{e}$ ), then the outer radius is limited to the external radius (i.e. $\left.R_{o}=R_{e}\right)$.

To determine the power loss, the viscous drag torque viscous medium that intercedes the wetted area of the clutch disc needs to be determined. Therefore, the flow in the circumferential direction of the disc face requires consideration. It is noteworthy that wet clutch friction discs typically contain radial grooves to allow oil flow and heat transfer throughout the engagement process. These grooves provide a variation in the shear stress in the circumferential direction as: $\tau(r)=\frac{\left|\Omega_{2}-\Omega_{1}\right| \eta r}{h}$. Therefore, the shear stress is determined for regions with and without the presence of grooves. In this study radial grooves of fixed width are considered. Therefore, the total groove width would be constant at any radial position: $g_{w}(r)=n w$, where $n$ is the number of grooves and $w$ is the individual groove width. Hence, the drag torque at any radial position becomes, for the case investigated here $g_{w}(r)$ is a constant.:

$T(r)=\left|\Omega_{2}-\Omega_{1}\right| \eta r^{2}\left[\frac{\left(2 \pi r-g_{w}(r)\right)}{h_{p}}+\frac{g_{w}(r)}{h_{g}}\right]$

By integrating equation (33) between the limits of $R_{i}$ and $R_{o}$ the overall parasitic drag torque is obtained as:

$T=\left|\Omega_{2}-\Omega_{1}\right| \eta\left(\frac{\pi}{2 h_{p}}\left(R_{o}^{4}-R_{i}^{4}\right)+\frac{n w\left(R_{o}^{3}-R_{i}^{3}\right)\left(h_{p}-h_{g}\right)}{3 h_{p} h_{g}}\right)$

By multiplying the absolute difference in angular velocities of the mating disc pairs, the power loss from the interface is determined as: 
$P=\left|\Omega_{2}-\Omega_{1}\right|^{2} \eta\left(\frac{\pi}{2 h_{p}}\left(R_{o}^{4}-R_{i}^{4}\right)+\frac{n w\left(R_{o}^{3}-R_{i}^{3}\right)\left(h_{p}-h_{g}\right)}{3 h_{p} h_{g}}\right)$

This somewhat simplified approach followed to consider drag torque between two discs has some limitation. Therefore, great care should be taken when applying such an approach to complex groove geometries, such as inclined grooves, waffle or even atypical radial groove designs. The assumptions made here are for the purpose of arriving at a time-efficient simulation tool.

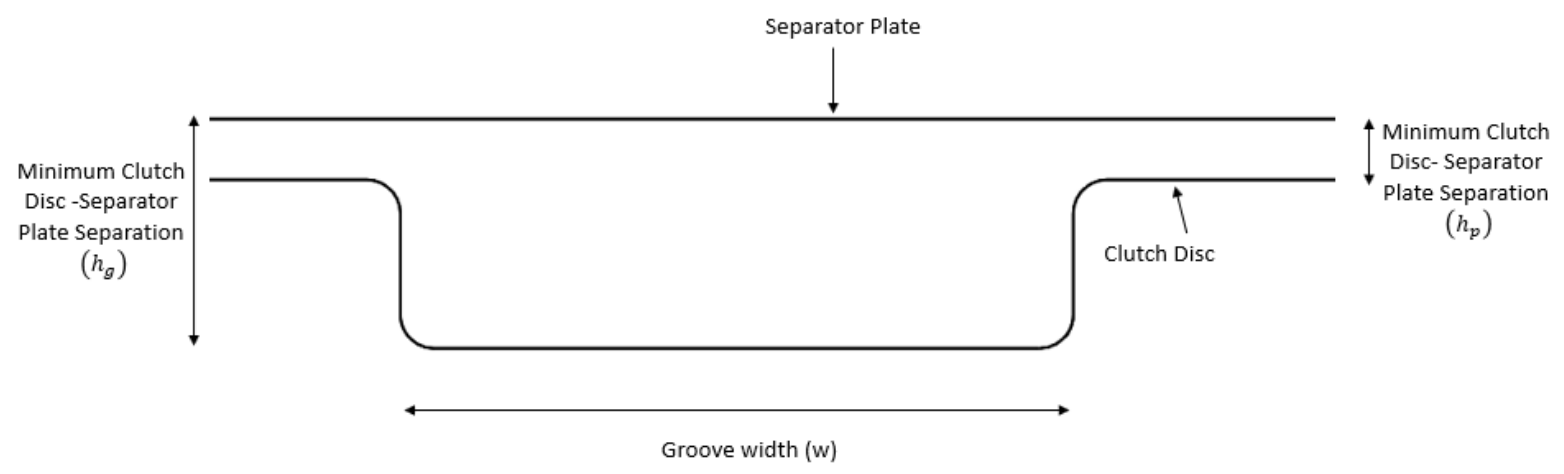

Figure 2: Cross section of a single groove geometry on clutch friction disc

\section{Validation}

The model is validated with experimental data available in open literature (Figure 2 and 3). Experimental measurements were reported by Neupert and Bartel [27], which were also used to validate their own model, including a multi-phase computational fluid dynamics model. The work in [27] is chosen because the input conditions are clearly stated and the inlet supply pressure was measured. As with the Neupert and Bartel [27] own validation an additional term is included to consider the torque on the internal section during the full film operation of the clutch disc. This is in additional torque given in equation (36) is in addition to the drag toque expressed by equation (34):

$T_{\text {int }}=\frac{\pi \eta\left|\Omega_{2}-\Omega_{1}\right| R_{i}^{4}}{2 h_{\text {int }}}$

The input data given by Neupert and Bartel [27] is provided in table 1. The measured inlet pressure [27] is shown in figure 3 and is dependent on various system specific characteristics. This pressure was measured at the centre of the clutch rather than at the inner radius of the clutch disc conjunction. This assumption can incur some small error as it does not account for any centrifugal flow in the inner disc region. The pressure at the free boundary is set to $800 \mathrm{~Pa}$ (gauge). This value is used to fit the rupture point of the model to the experimental data. The assumed pressure is reasonable when considering typical values for meniscus forces, and build-up of pressure in the clutch housing [29]. The running conditions are also described in [27], during which: $\left|\Omega_{2}\right|>\left|\Omega_{1}\right|$, as the separator plate remains stationary.

Table 1: Input Parameters

\begin{tabular}{lcc}
\hline Parameter & Value & Unit \\
\hline Viscosity at $30^{\circ} \mathrm{C}(\eta)$ & 0.043 & Pa.s
\end{tabular}


Density at $30^{\circ} \mathrm{C}(\rho)$

Inner Radius $\left(R_{i}\right)$

External Radius $\left(R_{e}\right)$

Pressure inner (gauge)

Pressure outer (gauge)

Minimum separation $\left(h_{p}\right)$

Groove-separator plate separation $\left(h_{g}\right)$

Internal section separation $\left(h_{\text {int }}\right)$

Groove Width(w)

Number of Grooves $(\mathrm{n})$

Angular velocity $\left(\Omega_{2}\right)$

$\begin{array}{cc}835 & \mathrm{~kg} / \mathrm{m}^{3} \\ 0.0706 & \mathrm{~m} \\ 0.0840 & \mathrm{~m} \\ 3000 \text { to }-200[26] & \mathrm{Pa} \\ 800 & \mathrm{~Pa} \\ 250 & \mu \mathrm{m} \\ 850 & \mu \mathrm{m} \\ 1.4 & \mathrm{~mm} \\ 1.5 & \mathrm{~mm} \\ 60 & - \\ 0-3000 & \mathrm{rpm}\end{array}$

The comparison between the current model's predictions and the reported measurements in [27] (figure 3) show good agreement in magnitude and shape of the variation of drag torque characteristics. The fluctuation in predicted torque at $1200 \mathrm{rpm}$ is due to a variation in the experimentally measured inlet pressure [27]. Figure 3 shows the accuracy of torque measurements of Neupert and Bartel [27].

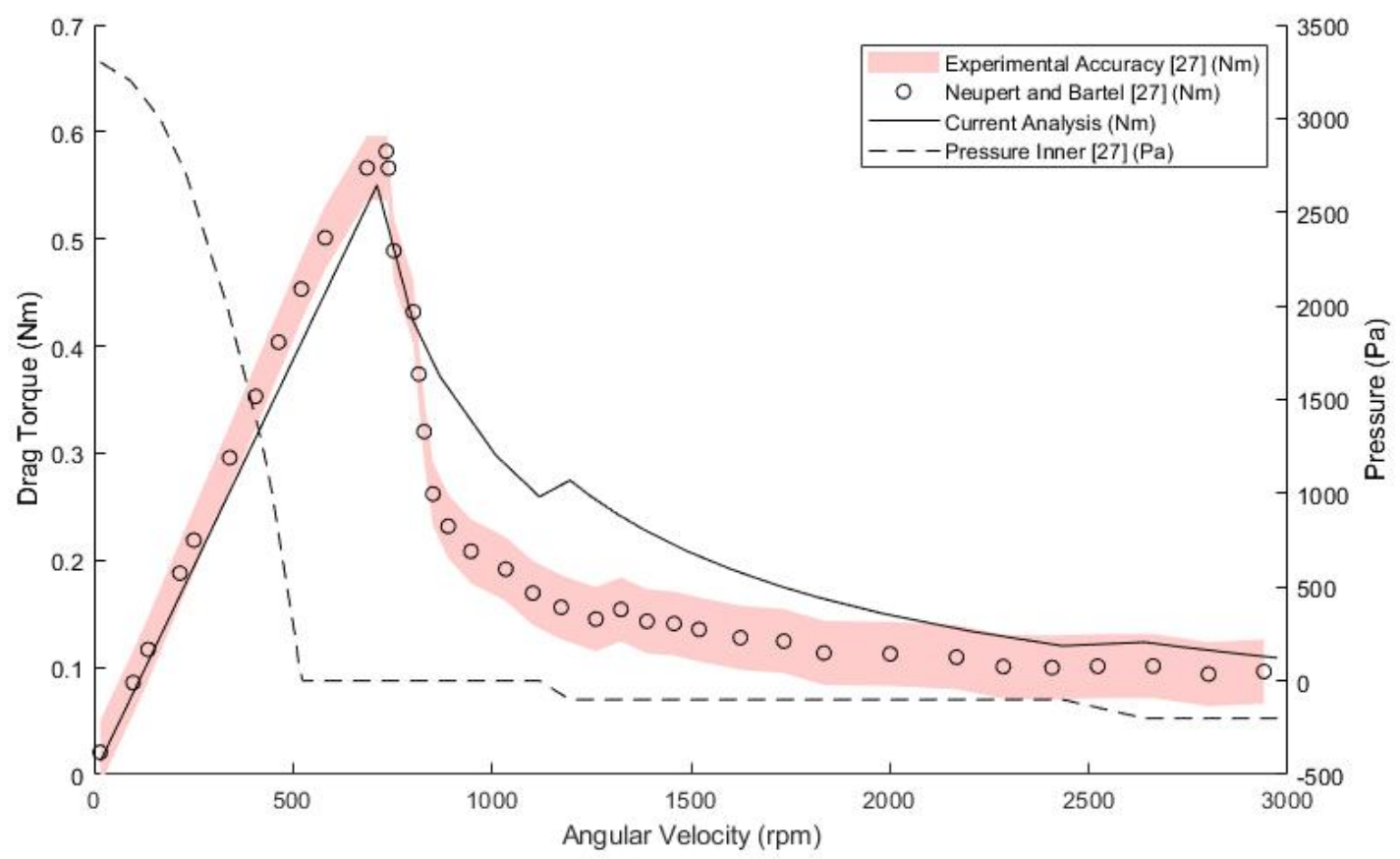

Figure 3: Validation of the separation part of the model with measurements of Neupert and Bartel [26].

A validation of the second part of the model (equation (34)) is conducted through comparison with experimental measurements of Aphale et al [28]. The operational conditions employed for this particular set of results (figure 4) do not induce lubricant film rupture. The parameters given in the experimental work are listed in table 2.

Table 2: Input Parameters from Aphale et al [28]
Value

Unit 


\begin{tabular}{lcc}
\hline Viscosity $(\eta)$ & 0.48 & Pa.s \\
Inner Radius $\left(R_{i}\right)$ & 0.0720 & $\mathrm{~m}$ \\
External Radius $\left(R_{e}\right)$ & 0.0813 & $\mathrm{~m}$ \\
Minimum separation $\left(h_{p}\right)$ & 200 & $\mu \mathrm{m}$ \\
Groove-stator separation $\left(h_{g}\right)$ & $400 \& 600$ & $\mu \mathrm{m}$ \\
Angular velocity $\left(\Omega_{2}\right)$ & 500 & $\mathrm{rpm}$ \\
Groove Width $(\mathrm{w})$ & 2 & $\mathrm{~mm}$ \\
Number of Grooves(n) & $40-80$ & - \\
\hline
\end{tabular}

The results in figure 4 provide a comparison of the model preditions with the measurements of Aphale et al [28]. The shaded area indicates plus and minus one standard deviation of the experimental measurements [28].

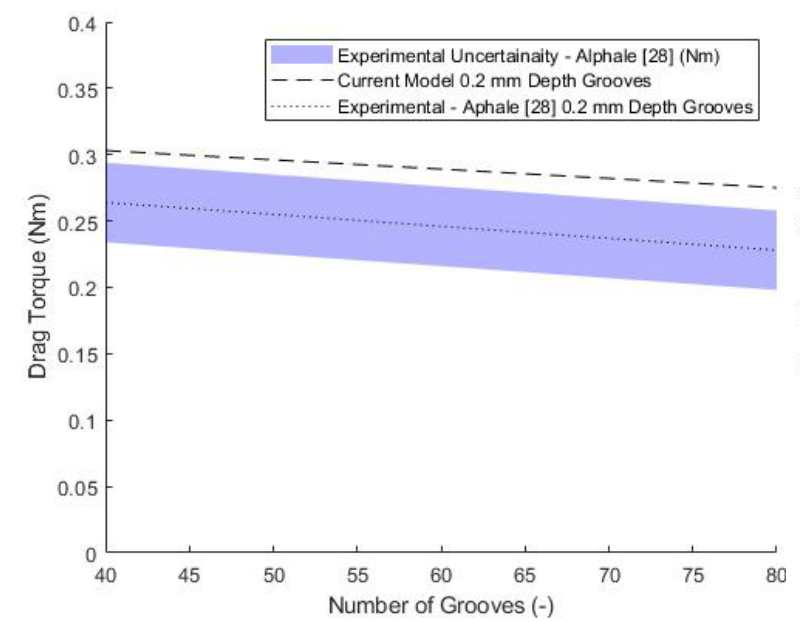

(a)

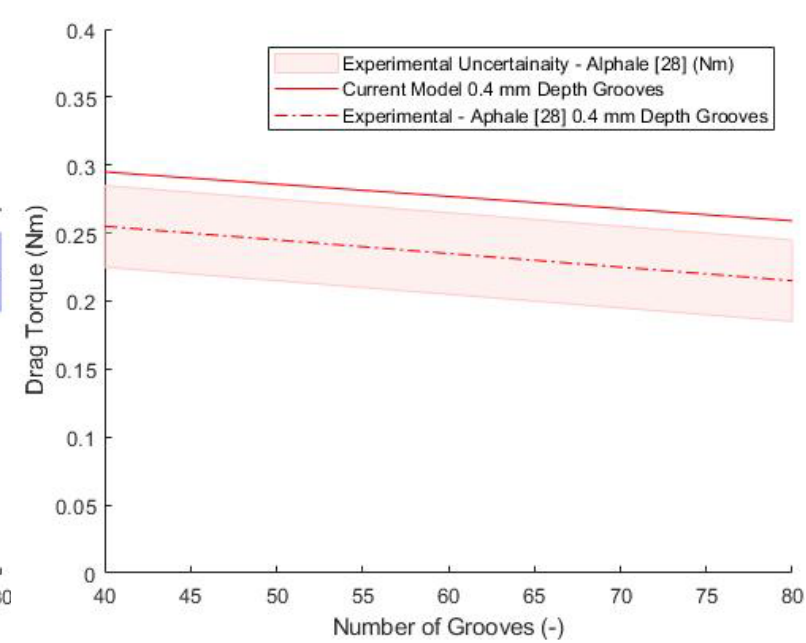

(b)

Figure 4: Validation of the separation predictions of the model with measurements of Alphale et al [28]

Reasonable agreement is figure 4 for both magnitude and trends for predictions for a range of numbers of grooves and their depths with the measurements in [28]. The over-predications can be accounted for as the result of small changes in lubricant temperature due to viscous shear heating or presence of lubricant aeration.

\section{Results and Discussion}

The results presented in the following section show the disengaged drag toque during co-directional and counter-directional rotations of wet clutch discs at varying relative disc speeds. The input parameters used for the model are the same as those employed for model validation above (i.e. table 1). In lieu of experimentally measured pressure differentials between the inner and outer radii for a full range of counter and co-directional rotating conditions investigated in the following analysis a fixed value for $p_{o}-p_{i}=1.1(\mathrm{kpa})$ is used.

The variation of drag torque at various co-directional and counter-directional rotations is shown in figure 5 . The results are for a single friction and separator disc interface (i.e. not the additional values 
stated in equation (36), which includes other losses taken into account in the model validation exercise). The largest drag torque occurs during counter-directional rotation in the top left and bottom right quadrants. This is because in these locations high shear rates: $\left(\frac{\left|\Omega_{2}-\Omega_{1}\right|}{h}\right)$ occur as a result of counter directional rotation. However, the drag torque does not increase monotonously with shear rate as it is also affected by the area over which a coherent film of lubricant is sustained in the disc pair's conjunction. At higher combined angular velocities in counter-directional rotation the extent of fluid film's outer radius recedes. This reduces the area over which viscous shear stress acts.

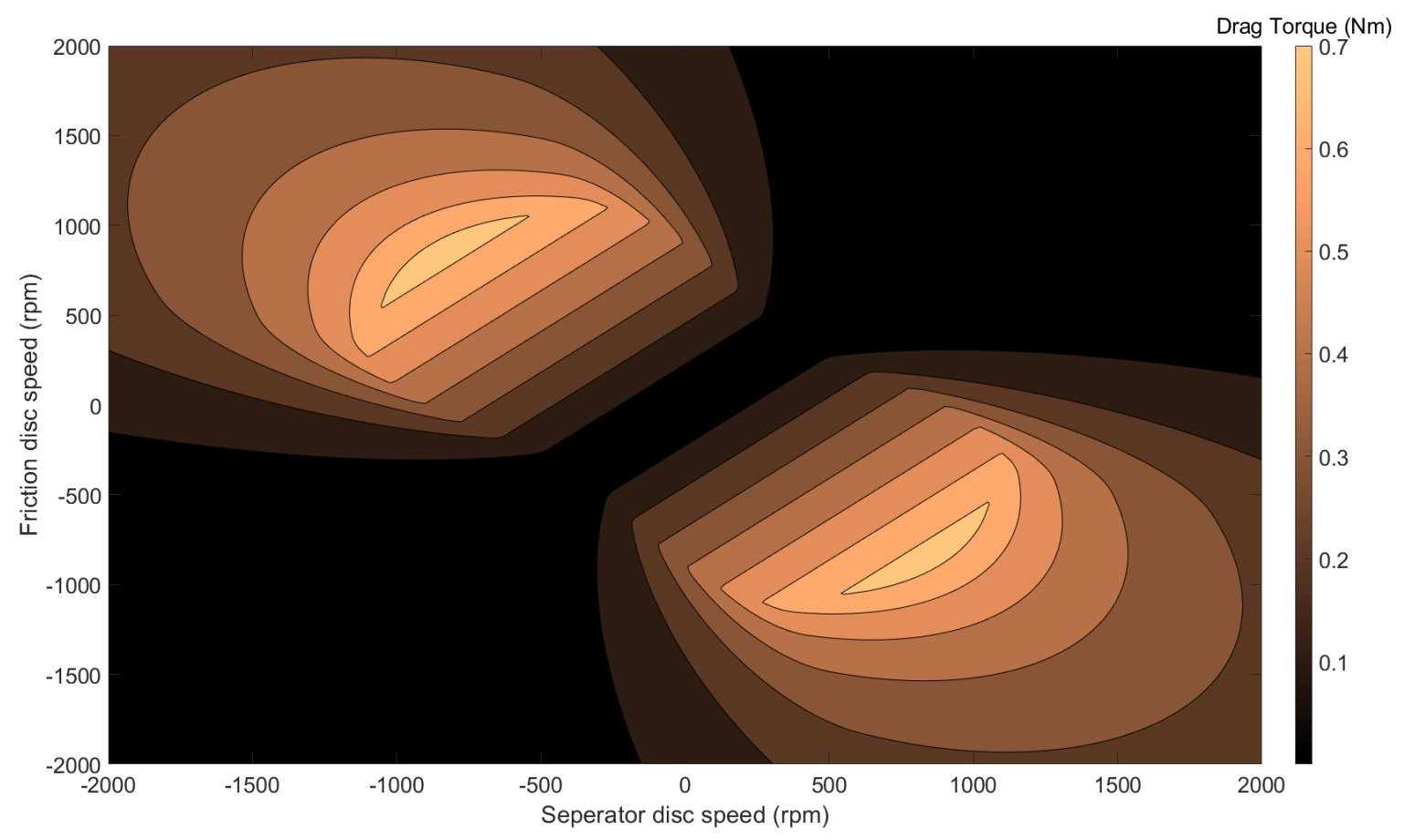

Figure 5: Drag torque under varying co-rotation and counter rotation conditions

The power loss created by the hydrodynamic drag friction torque (shown in figure 5 ) is presented in figure 6 . The results show a similar trend to the friction torque characteristics itself. For the conditions investigated in this paper, representative of a vehicular cold start condition (at $30^{\circ} \mathrm{C}$ ), the power losses for a single facing pair are shown to be 120 Watts under the least favourable kinematic operating conditions. This amounts to a considerable parasitic loss when it is considered that wet clutch packs typically consist of multiple friction and separator discs. 


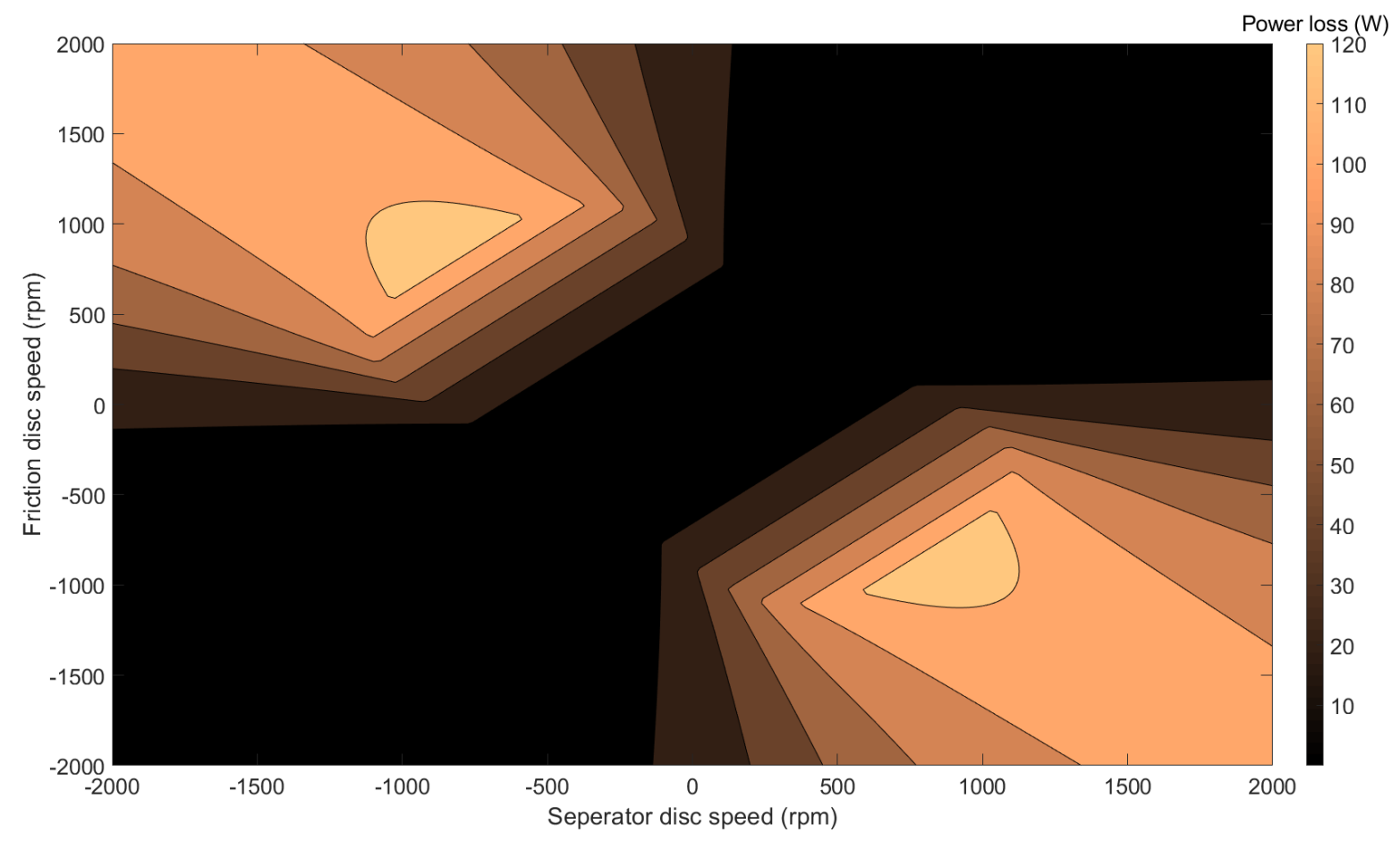

Figure 6: Power loss under varying co-rotation and counter rotation conditions

The radial location of lubricant film rupture can be clearly observed in figure 7. In slow speed operation the outer radial boundary at the lubricant film rupture is located at the outer edge of the discs (i.e. $0.084 \mathrm{~m}$ ). Under this condition the plates are fully wetted and the maximum area of lubricant film shear occurs. With a progressively increasing speed, beyond a critical combination of friction disc and separator disc speeds, the lubricant film begins to separate. This can be seen during co-directional rotation. The separation occurs at lower combined angular velocity combinations. During codirectional rotation the radial extent of lubricant film separation position rapidly approaches the inner radius of the disc with any further small increase in the angular velocity. This explains the drag torque behaviour and parasitic power loss observed in figures 5 and 6 . 


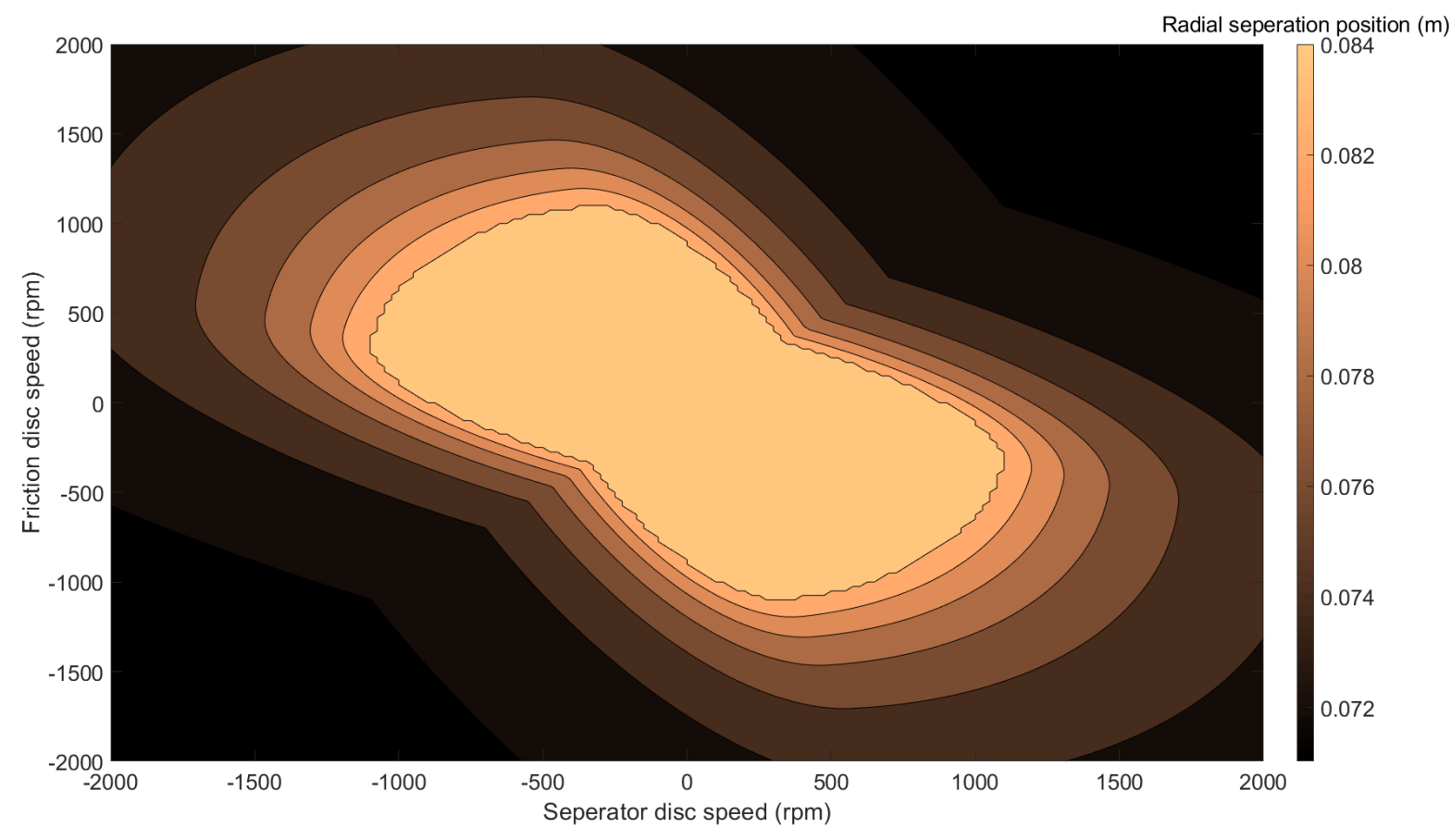

Figure 7: Radial location of film rupture position

During co-directional rotation or when either disc is stationary the location of separation boundary is always on the slower of the two moving surfaces. In the case of counter-directional rotation the lubricant film rupture depends on the ratio of the two angular velocities and can occur at any axial location $\left(0 \leq Z_{s} \leq h\right)$. The dimensionless axial location of film rupture $\left(z_{s} / h\right)$ can be observed clearly in figure 8 . In this figure unity indicates occurrence of separation at the fiction disc, whilst a null value (zero) indicates that separation takes place on the separator disc. Values between zero and one show the fractional position across the film height at which incipient reverse flow would ensue. During these conditions the lubricant is swept away by both the counter-rotating surfaces. The quantity of oil swept away by each surface may have an influence on the heat transfer and cooling at the outer regions of the discs. This is the primary purpose of lubricant supply to these types of conjunction. 


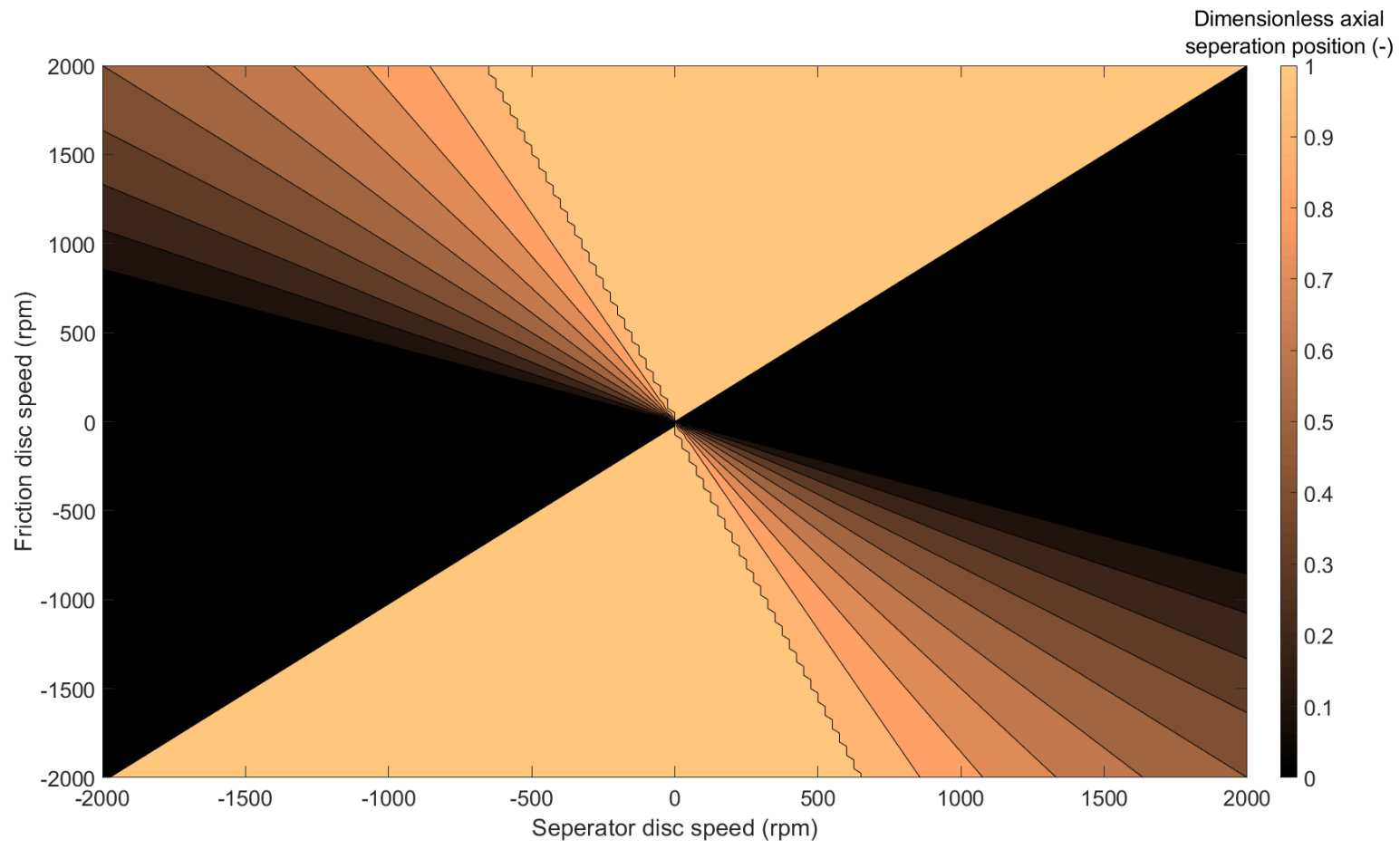

Figure 8: Film rupture axial location graph of $z_{s} / h$ with varying operating speed condition

\section{Conclusion}

A new analytical implicit method for disengaged wet clutch drag losses is presented. The model takes into account lubricant film rupture through the mechanism of oil film separation, in line with observations in practice, reported in the open literature. The model can consider the various disengaged wet clutch operating conditions, which occur in practice such as co-directional and counter-directional disc pair rotations. The results highlight the operating conditions under which significant parasitic drag losses occur. In addition, the results also show that during co- directional rotation of discs there is little drag torque as a result of significant oil film separation that can occur even under low angular velocities.

\section{Acknowledgements}

This work was supported by the J.C. Bamford Excavators and Innovate UK.

\section{References}

[1] Berger, E.J., Sadeghi, F. and Krousgrill, C.M., 1997. "Torque transmission characteristics of automatic transmission wet clutches: experimental results and numerical comparison", Tribology Transactions, 40(4), pp.539-548.

[2] Davis, C.L., Sadeghi, F. and Krousgrill, C.M., 2000. "A simplified approach to modeling thermal effects in wet clutch engagement: analytical and experimental comparison", Journal of Tribology, 122(1), pp.110-118. 
[3] Walker, P.D., Zhang, N., Tamba, R. and Fitzgerald, S., 2011. "Simulations of drag torque affecting synchronisers in a dual clutch transmission", Japan Journal of industrial and applied mathematics, 28(1), pp.119-140.

[4] Marklund, P., 2008. "Wet clutch tribological performance optimization methods (Doctoral dissertation", Luleå tekniska universitet), Luleå, Sweden.

[5] Pahlovy, S., Mahmud, S.F., Kubota, M., Ogawa, M. and Takakura, N., 2014. "Multiphase drag modeling for prediction of the drag torque characteristics in disengaged wet clutches", SAE International Journal of Commercial Vehicles, 7(2014-01-2333), pp.441-447.

[6] Iqbal, S., Al-Bender, F., Pluymers, B. and Desmet, W., 2014. "Model for predicting drag torque in open multi-disks wet clutches", Journal of Fluids Engineering, 136(2), p.021103.

[7] Yuan, Y., Liu, E.A., Hill, J. and Zou, Q., 2007. "An improved hydrodynamic model for open wet transmission clutches", Journal of Fluids Engineering, 129(3), pp.333-337.

[8] Morris, N., Davies, J., Leighton, M., King, P.D. and Rahnejat, H., 2019. "Oil film separation and drag torque in disengaged wet brakes". Proceedings of the Institution of Mechanical Engineers, Part D: Journal of Automobile Engineering, p.0954407019844358.

[9] Swift, H.W., 1932. "The stability of lubricating films in journal bearings", Journal of the Institution of Civil Engineers, 233(1):267.

[10] Stieber, W., 1933. “Dus Schwimmlager. Verein Deutscher”, Berlin: Ingenieurre.

[11] Mahmud, S.F., Pahlovy, S.A., Kubota, M., Ogawa, M. and Takakura, N., 2015. "Multi-Phase Simulation for Predicting Better Groove Pattern of the Clutch Disk for Low Drag Torque(No. 2015-011977)", SAE Technical Paper.

[12] Jammulamadaka, A.K. and Gaokar, P., 2011. "Spin Loss Computation for Open Clutch Using CFD”, SAE International Journal of Engines, 4(2011-01-1238), pp.1536-1544.

[13] Yuan, Y., Attibele, P. and Dong, Y., 2003. "CFD simulation of the flows within disengaged wet clutches of an automatic transmission (No. 2003-01-0320)", SAE Technical Paper.

[14] Yuan, S., Guo, K., Hu, J. and Peng, Z., 2010. "Study on aeration for disengaged wet clutches using a two-phase flow model", Journal of Fluids Engineering, 132(11), p.111304.

[15] Zweig, J.E. and Sneck, H.J., 1976. "Two-fluid flow between rotating annular disks", Journal of Lubrication Technology, 98(2), pp.214-222.

[16] Jibin, H., Zengxiong, P. and Chao, W., 2012. "Experimental research on drag torque for singleplate wet clutch", Journal of Tribology, 134(1), p.014502.

[17] Aphale, C.R., Schultz, W.W. and Ceccio, S.L., 2011. "Aeration in Lubrication With Application to Drag Torque Reduction", Journal of Tribology, 133(3), p.031701.

[18] Coombs, J.A. and Dowson, D., 1964, "Paper 12: An Experimental Investigation of the Effects of Lubricant Inertia in a Hydrostatic Thrust Bearing", In Proceedings of the Institution of Mechanical 
Engineers, Conference Proceedings (Vol. 179, No. 10, pp. 96-114). Sage UK: London, England: SAGE Publications.

[19] Brunetière, N., Tournerie, B. and Fre, J., 2002. "Influence of fluid flow regime on performances of non-contacting liquid face seals", Journal of Tribology, 124(3), pp.515-523.

[20] Hou, S., Hu, J. and Peng, Z., 2017. "Experimental Investigation on Unstable Vibration Characteristics of Plates and Drag Torque in Open Multiplate Wet Clutch at High Circumferential Speed", Journal of Fluids Engineering, 139(11), pp.111103.

[21] Birkhoff, G. and Hays, D.F., 1963. "Free boundaries in partial lubrication", Studies in Applied Mathematics, 42(1-4), pp.126-138.

[22] Dowson, D., 1961. "Inertia effects in hydrostatic thrust bearings", Journal of Basic Engineering, 83(2), pp.227-234.

[23] Coyne, J.C. and Elrod, H.G., 1970. "Conditions for the rupture of a lubricating film. Part I: theoretical model", Journal of Lubrication Technology, 92(3), pp.451-456.

[24] Coyne, J.C. and Elrod, H.G., 1971. "Conditions for the rupture of a lubricating film-part ii: New boundary conditions for Reynolds equation", Journal of Lubrication Technology, 93(1), pp.156-167.

[25] Hopkins, M.R., 1957. "Viscous flow between rotating cylinders and a sheet moving between them", British Journal of Applied Physics, 8(11), p.442.

[26] Dowson, D. and Taylor, C.M., 1979. "Cavitation in bearings", Annual Review of Fluid Mechanics, 11(1), pp.35-65.

[27] Neupert, T. and Bartel, D., 2019. "High-resolution 3D CFD multiphase simulation of the flow and the drag torque of wet clutch discs considering free surfaces", Tribology International, 129, pp.283296.

[28] Aphale, C.R., Cho, J., Schultz, W.W., Ceccio, S.L., Yoshioka, T. and Hiraki, H., 2006. "Modeling and parametric study of torque in open clutch plates", Journal of tribology, 128(2), pp.422-430.

[29] Pahlovy, S.A., Mahmud, S.F., Kubota, M., Ogawa, M. and Takakura, N., 2016. "New development of a gas cavitation model for evaluation of drag torque characteristics in disengaged wet clutches", SAE International Journal of Engines, 9(3), pp.1910-1915. 